\title{
Lumen
}

Selected Proceedings from the Canadian Society for Eighteenth-Century Studies

\section{Sade, noir et blanc : Afrique et Africains dans Aline et Valcour}

\section{Catherine Gallouët}

Volume 24, 2005

Indigenes and Exoticism

Indigènes et exotisme

URI : https://id.erudit.org/iderudit/1012175ar

DOI : https://doi.org/10.7202/1012175ar

Aller au sommaire du numéro

Éditeur(s)

Canadian Society for Eighteenth-Century Studies / Société canadienne d'étude du dix-huitième siècle

\section{ISSN}

1209-3696 (imprimé)

1927-8284 (numérique)

Découvrir la revue

Citer cet article

Gallouët, C. (2005). Sade, noir et blanc : Afrique et Africains dans Aline et

Valcour. Lumen, 24, 65-78. https://doi.org/10.7202/1012175ar

Copyright (c) Canadian Society for Eighteenth-Century Studies / Sociéte canadienne d'étude du dix-huitième siècle, 2005
Ce document est protégé par la loi sur le droit d'auteur. L'utilisation des services d'Érudit (y compris la reproduction) est assujettie à sa politique d'utilisation que vous pouvez consulter en ligne.

https://apropos.erudit.org/fr/usagers/politique-dutilisation/ 


\section{Sade, noir et blanc: Afrique et Africains dans Aline et Valcour}

Aline et Valcour $^{2}$, roman-fleuve épistolaire ${ }^{3}$, écrit à la Bastille juste avant la Révolution, occupe une place privilégiée dans l'œuvre de Sade. On sait le soin qu'apporta l'auteur à sa composition et même à sa préservation. C'est que «Sade entend faire de ce roman [...] son chef-d'œuvre, avec tout le soin, le poli et l'équilibre qu'implique ce terme ${ }^{4} »$. Cet ouvrage, quoique fort peu analysé par la critique sadienne, est aux yeux de son auteur aussi précieux, aussi fondamental, que Les Cent vingt journées de Sodome. Dans une démarche délibérée, Sade exploite la forme des grands romans, et «ne s'y prive d'aucune des ressources du genre, fussent-elles les plus extravagantes ${ }^{5} »$. Le texte du roman sentimental foisonne, se

1 Certaines parties de ce travail ont été présentées au Colloque International Sade à Charleston (N.C.) en mars 2003, et au Congrès International des Études Francophones à la Nouvelle Orléans en juin 2003. La recherche a été effectuée à la Bibliothèque Nationale de France, avec le soutien de Hobart et William Smith Colleges, que je remercie ici.

2 Le titre complet du roman est Aline et Valcour, ou le roman philosophique. Écrit à la Bastille un an avant la Révolution de France. Nous utilisons l'édition de Sade, CEuvres, volume I, établie par Michel Delon, Paris, Gallimard, coll. «Bibliothèque de la Pléiade», 1990.

3 Comme l'écrit Sade lui-même plaidant la cause de son roman aux autorités, "Cet ouvrage volumineux est le fruit de plusieurs années de veille ». Cité par Jean M. Goulemot dans l'introduction de son édition d'Aline et Valcour ou le roman philosophique, Paris, Librairie Générale Française, «Le Livre de Poche classique», 1994, p.10.

4 Jean Fabre, «Préface», CEuvres complètes du marquis de Sade, Tome quatrième, Paris, Au Cercle du Livre Précieux, p. XII. Voir aussi la Notice de Michel Delon, dans Sade, CEuvres, p. 1196-1213.

5 Annie Le Brun, Soudain un bloc d'abîme, Sade, Paris, Pauvert, 1986, p. 171. Sade reste ancré dans la tradition qui nourrit le roman du XVII ${ }^{\mathrm{e}}$ et du XVIII ${ }^{\mathrm{e}}$ siècles, citant en particulier Théagène et Chariclée dans l'Idée sur les romans. Roger Mercier, quant à lui, pense que "c'est à la génération précédente que Sade s'apparente par ses conceptions littéraires", et en particulier à Prévost, dans "Sade et le thème des voyages dans Aline et Valcour», Dix-Huitième Siècle, 1, 1969, p. 337. 
dispersant avec les récits de voyages lointains qui assimilent, eux aussi, les éléments les plus variés du genre : "Sade opère [...] une synthèse au niveau thématique et, également, au niveau des genres romanesques ${ }^{6}{ }^{6}$. Derrière l'histoire sentimentale de l'amour persécuté d'Aline et Valcour, annoncée par le titre, une autre histoire, celle de Sainville et Léonore, envahit le roman dont elle occupe le centre et la plus grande partie. Cette histoire est en fait un double récit, celui des voyages que les protagonistes, chacun lancé à la recherche de l'autre, font à travers le monde. En vrai romancier du $\mathrm{XVIII}^{\mathrm{e}}$ siècle, Sade, prospecteur de la forme imaginaire, fait naviguer son texte entre le roman sentimental et la relation quasi anthropologique, fusionnant dans les voyages de Sainville et Léonore le domaine de l'imaginaire avec celui du vraisemblable ${ }^{7}$. Aline et Valcour est autant une somme romanesque qu'une somme philosophique.

Les circonstances de ce double récit méritent qu'on s'y arrête. Les deux personnages viennent d'arriver de façon quelque peu rocambolesque dans la propriété de Vertfeuille où sont réfugiées Aline et sa mère, Mme de Blamont, loin des persécutions de leur père et mari qui veut empêcher le mariage de la jeune fille avec son amant, Valcour. Sont aussi présents des amis fidèles, Déterville, M. de Beaulé, Mme de Senneval et sa fille Eugénie. Déterville tient son ami Valcour au courant des menus faits de cette société par des lettres quotidiennes. Les histoires de Sainville et Léonore font donc partie, dans leurs moindres détails, de cette correspondance, la matière même du roman; ce sont les lettres XXXV, pour l'histoire de Sainville, et XXXVIII, pour celle de Léonore, lettres absolument délirantes quand l'on sait qu'elles ont environ deux cent pages, et qu'elles sont toutes deux datées d'un certain 16 novembre ${ }^{8}$.

6 Cerstin Bauer-Funke, «Aline et Léonore ou les effets de la violence; violence et progrès dans Aline et Valcour ou le roman philosophique du marquis de Sade», dans Progrès et violence au XVIII ${ }^{\mathrm{e}}$ siècle, V. Cossy et D. Dawson (édit.), Paris, Honoré Champion, 2001, p. 168. L'excès romanesque sadien, y compris l'excès de violence, n'est pas sans rappeler celui de Marivaux dans Les Effets surprenants de la sympathie (1714). Pour la violence romanesque dans Marivaux, voir Catherine Gallouët, Marivaux, Journaux et fiction, Orléans, Éditions Paradigme, Coll. Références, 2001, p. 121-133.

7 La critique continue d'isoler les histoires de Sainville et Léonore du texte du roman. Sur les raisons de cette séparation, et sur ses conséquences critiques, voir Mladen Kozul, «Épistolarité et narration dans Aline et Valcour», SRAZ, XXXVI-XXXVII, 1991-92, p. 239-250.

8 Dans l'édition de M. Delon, la lettre XXXV sur Sainville est de 199 pages (525-724) dont 69 (550-609) sont consacrées à l'Afrique; la lettre sur Aline est de 217 pages dont 51 sont consacrées à l'Afrique (737-954). 
Le récit de Léonore révèle bientôt que celle-ci est en fait la fille perdue de Mme de Blamont : Aline et Léonore sont donc sæurs. Ainsi, que ce soit au niveau de la forme (récit oral intercalé, rapporté dans un échange de lettres), ou au niveau de la thématique (le retour de Léonore à la maison maternelle), l'histoire de Léonore et de Sainville est en quelque sorte retenue à l'intérieur d'un cadre narratif surdéterminé.

Pourquoi ce contrôle à l'intérieur d'un cadre sémantique rassurant? Le caractère hétérogène du récit de Léonore et de Sainville présente-t-il un danger pour la communauté réunie à Vertfeuille ? Récit de voyage, où mène-t-il ? - Dans un monde réputé absolument inconnu, proclame l'éditeur présumé de l'ouvrage :

Ceux qui aiment les voyages trouveront à se satisfaire, et l'on peut les assurer que rien n'est exact comme les deux différents tours du monde, faits en sens contraire par Sainville et par Léonore. Personne n'est encore parvenu au royaume de Butua, situé au centre de l'Afrique ; notre auteur seul a pénétré dans ces climats barbares ; ici ce n'est plus un roman, ce sont les notes d'un voyageur exact, instruit, et qui ne raconte que ce qu'il a vu. (387)

En définitive, les protagonistes ne font pas tant un tour du monde qu'un tour de l'Afrique, certes très long : Sainville arrive sur la côte atlantique du Maroc, et entre dans les terres par l'ouest, probablement à la hauteur du Sénégal actuel, et Léonore passe de Tripoli au Caire, et pénètre en Afrique en descendant le cours du Nil. La double relation du continent africain entoure celui-ci de l'ouest et de l'est, et amène le lecteur en son cœur, le mystérieux royaume de Butua. Ces récits décrivent donc deux Afriques, l'Afrique de l'Ouest, c'est-à-dire l'Afrique noire, et l'Afrique de l'Est, autrement dit l'Afrique blanche. Ces deux notions, noir et blanc, se rejoignent lorsque les deux protagonistes se retrouvent, à l'insu l'un de l'autre, à Butua. L'éditeur insiste sur l'importance de cet épisode : «Le lecteur qui prendrait ceci pour un de ces épisodes placé sans motif, et qu'on peut lire ou passer à volonté, commettrait une faute bien lourde» (525). Dans ce contexte, l'Afrique acquiert une importance particulière. Elle est le centre vers lequel convergent tous les voyages de découverte ; présence incontournable, elle occupe, littéralement, le centre du roman Aline et Valcour. Sainville et Léonore y pénètrent par des voies opposées, ou plutôt, pénètrent la masse territoriale de l'Afrique. Ces pénétrations procèdent d'un même parcours, d'une même démarche ; elles mènent à ce qu'il y a de plus inconnu, de plus intérieur, à ce qui se cache au plus profond du continent le plus sombre. L'Afrique est bien le lieu de révélation de «tout ce qu'il a y a de plus affreux [...] dans la nature» (387). L'aboutissement de longs voyages, Butua devient ainsi le centre du monde, le cour des ténèbres. 
Notre travail propose donc d'examiner l'épisode africain d'Aline et Valcour, en insistant plus particulièrement sur Butua vers lequel tout semble converger'. Mais peut-on parler d'épisode africain dans un roman qui se double et se dédouble, dans une série vertigineuse de parallèles et de contrastes, de correspondances et d'oppositions? En fait, ce système binaire, dont la complexité effare, éclaire aussi le sens du roman : "Aucune affirmation ne va sans son contraire, aucune vérité ne peut être définie de manière statique. La description formelle d'Aline et Valcour engage la signification philosophique du roman ${ }^{10}{ }$. Ainsi, l'épisode africain change la portée philosophique du roman en même temps qu'elle en change la géographie : suivi pour Sainville par l'île de Tamoé, l'opposé exact du royaume de Butua, et pour les deux protagonistes par le récit des horreurs infligées par l'inquisition, cet épisode acquiert une nouvelle épaisseur, comme si son inquiétante étrangeté diminuait devant le chaos de l'inquisition, proche et familière.

L'Afrique de Léonore et Sainville est une Afrique familière aux lecteurs du roman de Sade. Ils y retrouvent, magnifiés, tous les stéréotypes du texte "africaniste» ${ }^{11} \mathrm{du} X \mathrm{XVII}{ }^{\mathrm{e}}$ siècle. Sade reprend donc un texte ancien, mais en l'exploitant au-delà de toute logique, en multipliant les stéréotypes jusqu'à saturation, il produit quelque chose de nouveau, d'autant plus inquiétant que ces mêmes clichés se retrouvent au siècle suivant dans la rhétorique raciste du colonialisme européen en Afrique ${ }^{12}$. Le foisonnement du texte sadien cache de nombreuses stratégies discursives qui semblent toutes aboutir à des conclusions similaires. Le texte sadien préfigurerait-t-il le discours colonialiste ? L'outrance sadienne participerait-elle à cette transformation du cliché de l'Africain au XVIII ${ }^{e}$ siècle en texte propagandiste du colonialisme ? Cette question, trop vaste, ne saurait trouver réponse dans le cadre restreint du présent travail. On tentera toutefois d'interroger le texte dans le contexte de la représentation de l'Afrique du XVIII ${ }^{\mathrm{e}}$ siècle.

9 Cet essai tente d'analyser certaines composantes de la représentation de l'Africain et de l'Afrique chez Sade. Il conviendrait éventuellement de situer cet épisode dans le contexte $\mathrm{du}$ « roman philosophique» voulu par Sade.

10 M. Delon, «Notice», p. 1205 ; pour les «effets de symétrie» dans le roman entre l'Afrique et l'Europe, voir aussi p. 1205-07.

11 Nous reprenons ici le terme utilisé par Christopher L. Miller, Blank Darkness: Africanist Discourse in French, Chicago and London, The University of Chicago Press, 1985.

12 Sur cette rhétorique, voir Sven Lindqvist, «Exterminate all the Brutes». One Man's Odyssey into the Heart of Darkness and the Origins of European Genocide, translated from the Swedish by Joan Tate, New York, The New Press, 1996. 
L'Afrique des contemporains de Sade se conjugue selon plusieurs discours contradictoires. Quel que soit leur point de vue, les textes des voyageurs en Afrique, par ailleurs relativement rares, restent tous tributaires des textes portugais ou hollandais du XVI et du début du XVII siècles ${ }^{13}$. Dans les textes de Bernardin de Saint Pierre, comme le Voyage à l'Isle de France, l'Isle de Bourbon, au Cap de Bonne-Espérance (1773), et comme bien sûr Paul et Virginie (1788), l'Afrique est représentée comme un paradis terrestre, refuge aux maux de la civilisation. En contraste absolu avec cette Afrique édénique, le roman de J.L. Castilhon, Zingha, reine d'Angola (1769), qui se présente pourtant comme documentaire, évoque une Afrique cannibale et assoiffée de sang ${ }^{14}$. De l'Histoire naturelle de l'homme de Buffon (1749) à l'Encyclopédie, la continuité est certaine; les textes s'élaborent à partir d'un discours unique, toujours répété, enveloppé parfois dans un discours pseudo-scientifique autorisant les conjectures les plus folles ${ }^{15}$.

Techniquement, l'Afrique reste en grande partie inconnue. Les Français s'étaient établis au Sénégal dès 1624, alors que la Compagnie des Indes, qui voulait Madagascar, a dû se contenter de l'Isle Bourbon, l'Ile de la Réunion actuelle. En fait, les Français comme les Européens sont restés surtout sur les côtes ; il est vrai que le naturaliste Michel Adanson passa plus de quatre ans au Sénégal, mais son ouvrage, Familles des plantes (1763), porte surtout sur la flore, et ce n'est qu'en 1802 que Jean-Baptiste Léonard publie une chronique authentique basée sur l'expérience personnelle des lieux, Voyage au Sénégal ${ }^{16}$. C'est dire que l'Afrique reste un continent empreint de mystère. Peu d'explorateurs contemporains de Sade peuvent prétendre vraiment connaître les terri-

13 Voir par exemple Ernst Van Den Boogaart, «Colour prejudice and the yardstick of civility: the initial Dutch confrontation with Black Africans, 1590-1635», dans Racism and Colonialism, Robert Ross (éd.), La Haye, Martinus Nijhoff Publishers for Leiden University Press, 1982, p.34-54. Pour un résumé de la façon dont on conçoit l'Africain du Moyen Âge au XVIII", voir l'excellent article de Nicholas Hudson, "From "Nation" to "Race"; The Origin of Racial Classification in Eighteenth-Century Thought", Eighteenth-Century Studies, Volume 29, Number 3, Spring 1996, p. 247-264.

14 J.L. Castilhon, Zingha, reine d'Angola. Histoire africaine, édition établie et présentée par Patrick Graille et Laurent Quillerie, Bourges, Ganymede, 1993.

15 N. Hudson montre que le XVIII ${ }^{\mathrm{e}}$ tend à généraliser les attributs des non-Européens, alors que les textes précédents soulignaient leur diversité culturelle, p. 250-251.

16 Voyage au Sénégal ou Mémoires historiques, philosophiques et politiques sur les découvertes, les établissemens et le cominerce des Européens dans les mers de l'Océan atlantique, Paris, $\mathrm{H}$. Agasse, 1802. 
toires décrits dans son roman ; parmi eux, sans doute, l'aventurier et diplomate écossais James Bruce qui vécut en Afrique de 1757 à 1773 ; mais le récit de ses voyages, Travels to Discover the Source of the Nile, ne fut publié qu'en $1790^{17}$. Ainsi, malgré les assurances de l'éditeur sur l'exactitude des faits rapportés, l'Afrique de Sade est une Afrique imaginaire. Selon Michel Delon, les sources en sont connues : «Sade tire principalement son information sur l'Afrique du Voyageur français de l'abbé de la Porte (Paris, 1771-72, t. XIII, XIV et XV), de l'Histoire des deux Indes de Raynal (Amsterdam, 1770, souvent réédité), du roman de Jean-Louis Castilhon, Zingha, reined' Angola. Histoire africaine (1769), etc. ${ }^{18}{ }^{\prime}$ Alors que certains des territoires décrits par Sade sont relativement familiers (comme ceux couverts par le périple de Léonore), les autres, le royaume de Butua, du moins dans la représentation qui en est faite, sont des produits d'imagination où tous les fantasmes européens sur le noir continent se donnent libre cours.

Sainville ne part pas délibérément en Afrique, il y arrive, contraint et forcé : il échoue sur les côtes à la suite d'un naufrage alors qu'il est lancé à la poursuite de Léonore, enlevée à Venise. Son voyage n'est donc pas un voyage de découvertes; il reproduit la quête très traditionnelle de l'objet aimé pour laquelle son naufrage sur les côtes africaines n'est qu'un obstacle supplémentaire. Or dès son arrivée, Sainville, l'amant, est confronté à l'Afrique dans toute son horreur, une terre qui confirme ses pires terreurs. Sainville, caché dans un arbre, partagé entre la fascination et l'horreur, et alors qu'il vient à peine d'échouer sur le continent, assiste au festin rituel de guerriers Jagas consommant la chair de ceux qu'ils viennent de tuer en combat. Ce spectacle horrible le laisse sans mots, incapable de représenter ce qu'il voit : "Comment vous peindre ce qui se passa !» (551). Ces exclamations sont toutes rhétoriques et ne l'empêchent pas de continuer son récit en insistant sur la «voracité», l'«espèce de rage et de frénésie» qui marquent ces «exécrables cérémonies» et qui doivent provoquer «l'épouvante et l'horreur» (552).

En fait, il s'avère que le phénomène du cannibalisme affecte toutes les perceptions de Sainville. Ainsi, avant même de commencer sa description de Butua, Sainville conditionne son auditoire en surdéterminant son

17 Le récit de Bruce et celui de Sade comportent des analogies pour le moins étonnantes. Bruce avait séjourné à Paris pour un traitement médical lors de son retour d'Afrique et avant son arrivée en Angleterre (voir Miles Bredin, Pale Abyssinian New York, Harper Collins Publishers, 2001). Sade avait-il entendu parler de ses exploits?

18 Michel Delon, p. 1258, et P. Graille, «Introduction» de J.L. Castilhon, Zingha, reine d'Angola. Histoire africaine, p. XXIII-XXIV. 
récit : "J'étais en un mot dans le royaume de Butua, habité par des peuples anthropophages, dont les mœurs et les cruautés surpassent en dépravation tout ce qui a été écrit et dit, jusqu'à présent, sur le compte des peuples les plus féroces» (554). Sainville semble être incapable de voir au delà de cet aspect de la culture du royaume de Butua : alors que, pour avoir la vie sauve, il accepte des pratiques qui devraient répugner à ce philosophe en puissance, telle que l'examen intime systématique de toutes les femmes qui sont promises au roi des lieux, il ne peut jamais accepter la logique du Portugais Sarmiento qui l'encourage à adopter les habitudes alimentaires du pays. Cette outrance au-delà de toute raison le fait «frémir», lui met «le cœur sur les lèvres» (561) ; pour Sarmiento il s'agit tout simplement d'un fait de société.

Sainville, lancé dans une quête amoureuse, devenu explorateur par la force des circonstances, ne change pas véritablement de registre ; il ne fait que passer du discours romanesque sentimental au discours romancé des récits de «vrais» voyages. N'est-il pas le seul Européen à avoir pénétré dans cet enfer ? Il se présente alors comme le conteur original de faits inédits, celui qui ramène la relation de terres jusque-là inconnues. Conscient de la nouveauté de son propos, et habile conteur, Sainville cajole son public, le faisant frémir à l'avance des horreurs qu'il promet de lui raconter :

Comme aucun Européen n'était parvenu dans cette partie ; que les Portugais n'y avaient point encore pénétré pour lors [...], comme il n'existe aucune relation de ces contrées, j'imagine que vous ne serez pas fâchés d'apprendre quelques détails sur la manière dont ces peuples se conduisent ; $j$ 'affaiblirai sans doute ce que cette relation pourra représenter d'indécent ; mais pour être vrai, je serai pourtant obligé quelquefois de révéler des horreurs qui vous révolteront. Comment pourrai-je autrement vous peindre le peuple le plus cruel et le plus dissolu de la terre? $(554)$

Aline est effarouchée par ces remarques et prête à quitter la pièce. On la retient fermement: «Ceci n'est pas une historiette indécente : monsieur ne va pas nous faire un roman ; c'est une partie de l'histoire humaine qu'il va peindre ; ce sont des développements de mœurs ; si vous voulez profiter de ces récits, si vous désirez y apprendre quelque chose, il faut donc qu'ils soient exacts» (id.). Il est vrai que le devoir d'exactitude scientifique s'impose. L'assemblée de Vertfeuille, à l'image de ses contemporains, ne sait pratiquement rien de l'Afrique dont Sainville va faire le récit. Un examen des cartes les plus courantes de l'époque montre que le plus souvent seules les côtes sont annotées ; le reste du territoire reste, pour ainsi dire, vierge. Quand une carte intérieure semble donner quelques détails, on s'aperçoit vite que ceux-ci sont de la plus pure 
fantaisie, qu'il s'agisse de détails géographiques ou d'animaux mythiques qui sont le résultat des spéculations de leur auteur ${ }^{19}$.

Toutefois, malgré la méconnaissance de l'Afrique au XVIII ${ }^{\mathrm{e}}$ siècle, on est persuadé d'une chose, qu'elle est anthropophage. Le texte cannibale s'est élaboré à partir des descriptions des cannibales amérindiens censés avoir été observés par Han Staden, prisonnier des Tupinamamba $\mathrm{d}^{\prime}$ Amérique latine lors d'un voyage avec les Portugais ${ }^{20}$. Selon W. Arens, ce récit, dont il semble qu'il n'a jamais été corroboré, est le premier de l'Europe moderne et fonde les récits cannibales «véridiques» qui ont contaminé l'imagination européenne à partir du $X V^{e}$ siècle. En fait, dès le Moyen Âge, le cannibalisme est posé comme la preuve même de la différence sauvage, et devient dès lors l'expression la plus irréductible de son altérité. Si ce discours est détourné par Montaigne qui oppose la férocité naturelle des cannibales à la sauvagerie des combattants des guerres de religion ${ }^{21}$, il est repris la plupart du temps comme épouvantail. Le discours européen sur l'autre se fonde sur son irréductibilité. L'autre, et par conséquent l'Africain, est donc cannibale ${ }^{22}$.

Le discours de Sainville sur l'Afrique se situe donc entre deux discours, le récit pseudo-scientifique du voyageur explorateur, dont le ton sera celui de l'observateur et du philosophe, et celui, imprégné des préjugés européens sur l'Afrique, du discours africaniste cannibale. Son récit reflète ces contradictions inhérentes. Conscient qu'il est le premier, Sainville veut montrer tous les aspects de la vie du royaume de Butua. Mais il se révèle incapable de «voir» l'autre en tant que sujet. Toutes les épithètes qu'il utilise pour décrire l'Africain renvoient une image stéréotypée unique : l'Africain, très noir de peau, est un barbare sanguinaire

19 Voir les cartes de J. N. Bellin, tirées de de la Carte Françoise de l'Océan Oriental Publiée Par Ordre de Mgr le Comte De Maurepas en 1740, Paris, 1740. Pour une carte fantaisiste, voir celle de J. Jansson, Aethioppia Superior vel Interior vulgo Abissinorum Sive Presbiteriioannis Imperium, Amsterdam, 1646, qui situe le royaume mythique du prêtre Jean entre le tropique du Cancer et la Mozambique, que l'on peut voir au site http://www.gracegalleries.com/Africa_Listings.htm.

20 Voir W. Arens, The Man-Eating Myth: Anthropology and Anthropophagy, Oxford, Oxford University Press, 1979, p. 22.

21 Montaigne, «Des Cannibales », dans Essais, Maurice Rat (édit.), livre I, Paris, Garnier Frères, 1962, p. 230-245.

22 Il ne s'agit pas ici d'entrer dans le débat engagé par W. Arens sur le cannibalisme. Il importe peu de savoir si oui ou non les Africains du siècle des Lumières étaient cannibales. Ce qui importe est que le XVIII ${ }^{\mathrm{e}}$ siècle n'en doutait pas, au point que toute représentation contemporaine de l'Africain y fait allusion. 
qui ne connaît que la cruauté, et dont toutes les actions offensent la raison. Sainville ne semble mû par aucune curiosité scientifique. Il ne sait qu'exprimer son dégoût : "Que d'infamies [...] ; toutes ces turpitudes me choquent au dernier point» (586). Malgré tout ce que lui en dit Sarmiento, Butua reste pour lui ce royaume « habité par des peuples anthropophages, dont les mœurs et les cruautés surpassent en dépravation tout ce qui a été écrit et dit, jusqu'à présent, sur le compte des peuples les plus féroces» (554). Quand il s'agit de signifier l'extrême degré de cruauté et de dépravation des habitants de Butua, ceux-ci sont immédiatement comparés aux Jagas, leurs voisins (583). C'est que les Jagas sont les prototypes mêmes des cannibales dans l'imagination européenne. Comme l'explique Béatrice Finck : «Although Butua is fictitious, the Jagas, or 'Giagues' were known cannibals in Sade's time» ${ }^{23}$. Ainsi, malgré les assurances de l'éditeur, et contrairement aux attentes de l'assemblée de Vertfeuille, le récit de Sainville n'inaugure pas un nouveau texte documentaire sur l'Afrique, mais continue de reproduire la tradition cannibale.

Notons, par ailleurs, que ce que Sainville donne comme son texte rapporte en fait les propos d'un autre personnage, Sarmiento, un vieux captif portugais, devenu ministre du roi de Butua. Le récit qu'entend l'assemblée réunie à Vertfeuille est en réalité celui de Sarmiento que Sainville reprend sans l'altérer. Sarmiento qui s'est chargé d'éduquer Sainville en lui donnant suffisamment de rudiments pour survivre lui explique systématiquement chacun des aspects du royaume de Butua, des habitudes sexuelles du roi à la finance, l'agriculture, le gouvernement, la servitude des femmes, la faune et la flore. Il justifie le cannibalisme en séparant logique et morale : «Ne comprends pas dans la corruption morale l'usage de manger de la chair humaine. Il est aussi simple de se nourrir d'un homme que d'un bœaf» (563). Sainville tente de protester, lui opposant des arguments éthiques, mais Sarmiento refuse d'entrer dans cette discussion; il ne veut être qu' «instituteur», et non pas «moraliste» (567). Son attitude est simple; puisqu'il s'agit de survivre, la raison dicte de s'adapter aux exigences des circonstances qui nous entourent : «Je ne ferais pas à Lisbonne ce que je ferais ici» (id.). Selon cette logique, les exigences de la vie varient d'une contrée à l'autre, et l'homme doit avant tout s'adapter à son environnement pour survivre. Cette relativité géographique, le produit d'une observation froide et

23 Béatrice Finck, «Sade and Cannibalism», L'Esprit créateur, volume 15, 4, Hiver 1975, 403-12, p. 406. En fait le royaume de Butua a prospéré à partir des années 1680 dans les plaines de Zimbabwe pendant près d'un siècle. 
scientifique de la réalité, devrait s'inscrire dans un discours neutre. Or il n'en est rien. Des remarques sur «la brutalité de ce despote» (559), «la férocité de ce peuple barbare» (564), montrent bien que le discours de Sarmiento n'échappe pas à l'origine de son orateur. En réalité, sa description insiste sur l'intempérance, la débauche, la cruauté, et souligne «le peu de morale», les "crimes multipliés», la crédulité et la superstition des habitants de Butua (id.). En définitive, malgré sa longue expérience à leurs côtés, les Africains restent opaques à son entendement : il admet ainsi qu'ils ont «quelques légères connaissances $\mathrm{d}^{\prime}$ astronomie, mais elles sont mêlées d'une si grande foule d'erreurs et de superstitions, qu'il est difficile d'y rien comprendre» (592). Malgré sa prétention au détachement moral, le discours de Sarmiento reflète une éthique européenne avérée ; de même, son texte sur Butua cache mal une méconnaissance fondamentale. Ses raisonnements ne sont pas basés sur l'expérience du terrain mais sur une interprétation ancrée dans la tradition africaniste, donc basée sur un savoir livresque. Ainsi, il s'autorise de la théorie des climats pour expliquer la sexualité masculine :

C'est à l'extrême chaleur de ce climat qu'il faut attribuer, sans doute, la corruption morale de ces peuples. [...] Mais ici il faut avouer que cette corruption est extrême : elle ne saurait être portée plus loin. Tous les désordres y sont communs, et tous y sont impunis ; un père ne met aucune espèce de différence entre ses filles, ses garçons, ses esclaves, ou ses femmes ; tous servent indistinctement ses débauches lascives. (580-81)

Dans le royaume de Butua, le libertinage est loi (586) ; Sarmiento, incapable de trouver une explication, produit de son observation personnelle et fruit de sa longue expérience à Butua, préfère expliquer ce fait de société par des théories sur l'autre issues de ses lectures. Ainsi le texte est surdéterminé à l'intérieur d'un système de représentation rassurant, tendance narrative que la narration au second degré de Sarmiento ne fait qu'accentuer. Il ne s'agit pas tant de rencontrer l'autre, mais bien d'en contrôler la représentation dans un discours maîtrisé.

Dans la mesure où le texte de Butua est déterminé exclusivement par Sarmiento (les indigènes n'ont pratiquement jamais la parole), les exclamations de Sainville devant celui-ci ajoutent encore un élément supplémentaire à l'élaboration du texte africain. A chaque nouvelle information apportée par Sarmiento, Sainville, horrifié, ne peut s'empêcher d'interrompre son interlocuteur : "Ce système anéantit toutes les vertus» (567) ; "Que d'infamies» (586) ; «Il n'est aucun principe de ces monstres que je ne mette au rang des plus affreux écarts de la raison humaine» (599). Ces hyperboles reproduisent celles communes au roman précieux et aux récits de voyages. Sainville se voulait anthro- 
pologue avant la lettre, mais la raison de ce philosophe romanesque ne se prête pas à l'observation de l'autre. Incapable de considérer l'autre en soi et de s'impliquer dans la culture qui l'entoure, il limite son regard à ce qu'il sait déjà de l'Afrique. En définitive, le langage lui fait défaut, et il a recours au discours d'un tiers pour exprimer une réalité dont le texte lui résiste. Ainsi, la représentation de l'Africain par Sainville et Sarmiento ne crée pas de nouveau texte, elle ajoute au texte africaniste du temps. Pris entre les exclamations de Sainville, inscrit dans une vision éthique résolument européenne, transmis dans une situation narrative qui en contrôle chaque aspect, déguisé par une démarche faussement anthropologique, l'épisode africain d'Aline et Valcour s'inscrit à l'intérieur d'un discours européen auquel il n'échappe absolument pas. La métaphore cannibale fait plus que reprendre le discours africaniste, elle le parfait et fait de l'Africain cannibale un produit de consommation européenne.

Rappelons encore une fois que, de tous les discours sur l'Afrique, le cannibalisme est celui sur lequel l'Europe s'est fixée, celui sur lequel elle a le plus fantasmé ; comme le résume Frantz Fanon «Le nègre est une bête, le nègre est mauvais, le nègre est méchant, le nègre est laid, [...] Le nègre va me manger ${ }^{24}$. C'est une attitude similaire qui est suggérée par la réaction de l'assemblée au récit de Sainville. On sait qu'Aline, effrayée par les promesses de Sainville de montrer toutes les horreurs de l'Afrique, avait presque quitté la compagnie et le lieu de la narration. Or, la compagnie apprend bientôt que Léonore a été parmi les femmes examinées incognito par Sainville pour servir l'appétit du roi de Butua :

«Oh, Dieu ! vous me faites frissonner, dit la présidente de Blamont, en interrompant Sainville ; quoi, monsieur, c'était Léonore ?... Quoi, madame, c'était vous ?... Et vous n'avez pas été... et vous ne fûtes pas mangée ?»

Toute la société ne put s'empêcher de rire de la vivacité naïve de la restriction plaisante de Mme de Blamont. (608-609)

Le danger d'être mangé est la première chose qui vient à l'esprit de la présidente. Sa remarque, involontaire, révèle l'obsession de tous pour le cannibalisme ; elle rapproche, par ailleurs, la consommation sexuelle (Léonore est examinée pour déterminer si elle a les qualités nécessaires pour le harem du roi), et l'anthropophagie ("vous ne fûtes pas mangée ?»), ce qui réduit considérablement la portée du danger anthro- 
pophage. Le frisson ressenti d'abord devant l'étrange mal contrôlé est remplacé par le rire qui montre bien que l'Afrique ne fait pas peur, parce qu'elle n'est pas véritablement autre. Alors même qu'il est censé être nouveau et terrible, le texte africain reste solidement maîtrisé à l'intérieur d'une sémantique qui ne quitte pas des normes que l'assemblée reconnaît sans effort. On comprend dès lors pourquoi on empêche Aline de quitter la pièce à l'annonce du récit cannibale : «Parlez, monsieur, parlez, que vos mots soient décents ; tout passe avec de bons termes ; soyez honnête et vrai, et surtout ne nous cachez rien ; ce qui vous est arrivé, ce que vous avez vu, nous paraît trop intéressant pour que nous en voulions rien perdre» (555). C'est qu'on est pressé d'en entendre la suite. On reconnaît dans cette avidité de l'auditoire devant la promesse d'un récit obscène un motif commun de la narration sadienne. Mais ici l'obscénité qu'attend l'auditoire n'est pas de nature sexuelle. L'assemblée, prête à avaler les moindres détails du récit avec la même avidité que les guerriers Jagas dévoraient leur butin de guerre, est bien prête à consommer l'Afrique : l'Afrique n'est pas tant le territoire des autres qu'un produit offert à la consommation, prêt à étre avalé par les honnêtes gens de l'assemblée, puis digéré et régurgité dans l'échange de lettres entre Déterville et Valcour. La logique du texte cannibale conduit à une «consommation» de l'Afrique qui va bien au-delà du plaisir du texte.

À écouter Sainville, il semble que tout se ramène au cannibalisme et que son expérience de l'Afrique se réduise à ce seul phénomène. On se souvient par ailleurs que lorsque Sarmiento propose à Sainville de partager son repas, Sainville s'y refuse absolument, préférant mourir plutôt que toucher à la chair humaine. Ne pouvant consommer la même viande que le peuple de Butua, Sainville doit donc se contenter de la chair dure et sèche des singes (561-62). Curieusement, Léonore aussi, qui après s'être échappée de Butua traverse le territoire des Jagas, survit en «ne mangeant que quelques singes tués à la chasse» (799). Ce choix de nourriture est pour le moins étrange : n'y a-t-il qu'un seul gibier en Afrique? Cela est d'autant plus curieux que lorsque le récit de Léonore avait évoqué plus tôt le singe africain, ils'agissait alors du singe roux $d^{\prime}$ 'Éthiopie «qui a le visage d'un homme, une très belle barbe blanche, et une voix semblable à celle d'une personne qui se plaint; il se tient communément sur les arbres, et ne s'apprivoise que très difficilement ; doué du même amour pour la liberté que l'homme, il dépérit et meurt dès qu'on l'enchaîne» (777). Ainsi le singe peut être à la fois presque humain et la viande principale des repas de Léonore en fuite, ce qui pourrait bien suggérer que Sainville et Léonore ne sont pas aussi incapables qu'ils le disent de digérer la chair humaine. Rappelons aussi qu'on associait alors dans les esprits les Africains aux singes, et qu'avec le discours cannibale, celui de l'animalité de l'Africain ou du sauvage 
$s^{\prime}$ imposait comme une évidence ${ }^{25}$. L'opposition binaire fondamentale qui divise celui qui mange son prochain (l'Africain cannibale) de celui qui s'y refuse (l'intrépide héros romanesque, explorateur malgré lui) est un des éléments qui forment ce que Philippe-Joseph Salazar dénomme «l'armature sémantique du discours de la race», discours qui offre «des signes repérables de reconnaissance [et affirme] la position de maîtrise du je européen, détenteur de la parole ${ }^{26}{ }^{2}$. Un autre élément est l'opposition homme/bête, animalité/ humanité. La consommation de l'élément africain (le singe) par l'Européen remplace les distinctions entre ces oppositions binaires par un motif suggérant une synthèse du je européen (Sainville et Léonore) avec l'autre africain (bestial). Cette consommation, cette ingestion, vide le motif du cannibalisme de toute son inquiétante étrangeté, affirme de façon concrète le contrôle absolu de l'autre, et annonce la mainmise de l'Européen sur son territoire, ce que le texte de Sade suggère sans équivoque : après s'être joué du roi de Butua, Léonore, agent des Portugais, livre le dernier territoire africain à résister à l'avance européenne à la colonisation. Appropriée dans le discours romanesque, l'Afrique devenue texte est prête à être absorbée territorialement.

L'Afrique est absente de l'Afrique de Sade : les Africains sont amputés d'être par les récits qui en sont faits, et ne vivent qu'à travers des fantasmes. Comme dans le texte de Joseph Conrad, elle est « Africa as setting and backdrop which eliminates the African as human factor. Africa as a metaphysical battlefield devoid of all recognizable humanity, into which the wandering European enters at his own peril ${ }^{27}$. » Dans le texte de Sade, l'Afrique est le ça qui reste déshumanisé, le territoire qui permet à Sainville et à Léonore de s'affirmer. Ainsi l'Afrique de Sade est, absolument, l'Afrique des Lumières, qui se prépare à l'expansion coloniale, l'Afrique des fantasmes blancs sur la négritude, sur la monstruosité cannibale de l'autre inconnu, ceux mêmes qui vont justifier les pires atrocités du colonialisme, comme si Sade, prophète, décelait les rouages secrets de la mentalité européenne dans le «noir continent». Sade, avant

25 Ainsi on attribuait la couleur de peau des Africains à un croisement avec les singes. Sur le débat sur l'origine simiesque des Africains, voir Léon Poliakov, «Le fantasme des êtres hybrides et la hiérarchie des races au XVII et XIX ${ }^{\mathrm{e}}$ siècles", dans Homme et bêtes; entretiens sur le racisme; actes du colloque tenu du 12 au 15 mai 1973 au Centre culturel international de Cerisy-la-Salle, Paris, Mouton, 1975, 167-181.

26 Philippe-Joseph Salazar, «Rhétorique de la race: imaginer l'autre au $18^{\mathrm{e}}$ siècle», French Studies in Southern Africa, 24, 1995, p. 88.

27 Chinua Achebe, «An Image of Africa: Racism in Conrad's Heart of Darkness», dans Hopes and Impediments. Selected Essays, New York, London, Anchor Books, 1990, p. 12. 
Conrad, "au cœur des ténèbres». Qu'on ne s'étonne plus dès lors de retrouver dans les textes colonisateurs du siècle suivant des échos des récits de Sainville et Léonore. L'outrance cannibale, devenue le texte identitaire de l'Afrique, est devenue la justification de l'entreprise coloniale : «Le portrait négatif permet de justifier à l'avance l'exploitation future qui sera faite au pré-civilisé dans sa terre natale et dans son corps par le Nouveau Héros bourgeois ${ }^{28} »$. Le texte sadien fait plus que reproduire le texte européen de l'Afrique noire dans la pensée européenne. L'outrance cannibale qui le marque annonce la rhétorique qui justifiera le colonialisme. Ainsi le blanc des cartes des voyageurs sera noirci, occupé par un nouveau texte, le discours colonial, où le contrôle sur l'autre se fait absolu.

\section{CATHERINE GALLOUËT}

Hobart \& William Smith Colleges

28 Lise Leibacher-Ouvrard, «Sauvages et utopies (1676-1715). L'exotisme-alibi», French Literature Series, Volume XIII, 1986, p. 5. Voir aussi Sven Lindqvist. 